\title{
On generalized Lebesgue-Ramanujan-Nagell equations
}

\author{
Attila Bérczes and István Pink
}

\begin{abstract}
We give a brief survey on some classical and recent results concerning the generalized Lebesgue-Ramanujan-Nagell equation. Moreover, we solve completely the equation $x^{2}+11^{a} 17^{b}=y^{n}$ in nonnegative integer unknowns with $n \geq 3$ and $\operatorname{gcd}(x, y)=1$.
\end{abstract}

\section{Generalized Ramanujan-Nagell equations}

Mixed polynomial-exponential equations are of classical and recent interest. One of the most famous equation of this type is the so-called RamanujanNagell equation, that is

$$
x^{2}+7=2^{n},
$$

where the unknowns $(x, n)$ are positive integers. In 1913 Ramanujan [76] conjectured that the above equation has only the solutions

$$
(x, n) \in\{(1,3),(3,4),(5,5),(11,7),(181,15)\} .
$$

Ljunggren posed the same problem in 1943 and Nagell [70] confirmed it in 1948. His proof in English was published in 1960 (see [72]). Subsequently, Chowla,

Key Words: Generalized Lebesgue-Ramanujan-Nagell equations; Primitive divisor theorem 2010 Mathematics Subject Classification: 11D41, 11D61

Received: January, 2014.

Accepted: February, 2014

This work was partially supported by the European Union and the European Social Fund through project Supercomputer, the national virtual lab (grant no.: TÁMOP-4.2.2.C11/1/KONV-2012-0010). The research was supported in part by the University of Debrecen, and by grants K100339 and NK104208 of the Hungarian National Foundation for Scientific Research. 
Lewis and Skolem [32] gave an other proof which uses Skolem's p-adic method (see also Hasse [43]).

In 1960, Apéry [3] showed that if $D>0$ is a given positive integer with $D \equiv 7$ $(\bmod 8), D \neq 7$ then the equation

$$
x^{2}+D=2^{n},
$$

has at most two positive integer solutions $(x, n)$. Note that equation $(2)$ possesses exactly two solutions if $D=23$ or $D=2^{k}-1$ for some $k \geq 4$. Indeed we have

$$
(x, n)= \begin{cases}(3,5),(45,11) & \text { if } D=23 \\ (1, k),\left(2^{k-1}-1,2 k-2\right) & \text { if } D=2^{k}-1,(k \geq 4) .\end{cases}
$$

Browkin and Schinzel [21] conjectured that apart from the values $D$ occurring in (3) equation (2) has at most one solution. By making a clever use of the hypergeometric method Beukers [17] confirmed the conjecture of Browkin and Schinzel.

Since then several extensions concerning equation (2) have been considered by many authors. Let $D_{1}, D_{2}, a, b$ be given non-zero integers with $D_{1} \geq 1, a \geq$ $1, b \geq 2$ and suppose that $\operatorname{gcd}\left(b, D_{1} D_{2}\right)=1$. Consider the equation

$$
D_{1} x^{2}+D_{2}=a b^{n},
$$

in positive integer unknowns $(x, n)$. If $D_{1}=1$ then equation (4) is usually called generalized Ramanujan-Nagell equation. (Note that if $\left(a, D_{1}, D_{2}, b\right)=$ $(1,1,7,2)$ then we get the original Ramanujan-Nagell equation). There are many results in the literature concerning upper bounds for the number of solutions of special cases of (4). Denote by $N\left(a, D_{1}, D_{2}, b\right)$ the number of solutions of (4).

\subsection{Upper bounds for $N\left(a, D_{1}, D_{2}, b\right)$ with $D_{2}>0$}

In 1960 Apéry [4] considered equation (4) with $\left(a, D_{1}, b\right)=(1,1, p)$ with $p$ an odd prime. He showed that for every fixed $\left(p, D_{2}\right)$ with $D_{2}>0$ and $p \nmid D_{2}$ the equation

$$
x^{2}+D_{2}=p^{n}
$$

has at most two solutions, that is $N\left(1,1, D_{2}, p\right) \leq 2$. The proof of the above result depends on elementary algebraic number theory. We mention that this result is sharp since for $\left(p, D_{2}\right)=(3,2)$ or $\left(p, D_{2}\right)=\left(4 k^{2}+1,3 k^{2}+1\right), k \geq 1$ equation (5) possesses exactly two solutions. Namely, we have

$$
(x, n)= \begin{cases}(1,1),(5,3) & \text { if }\left(p, D_{2}\right)=(3,2) \\ (k, 1),\left(8 k^{3}+3 k, 3\right) & \text { if }\left(p, D_{2}\right)=\left(4 k^{2}+1,3 k^{2}+1\right),(k \geq 1) .\end{cases}
$$


Thus there exist infinitely many pairs $\left(p, D_{2}\right)$ for which equation (5) has exactly two solutions.

Later several papers appeared improving the upper bound on the number of solutions of (5). For instance, Bender and Herzberg [11] proved that if $p$ is sufficiently large compared to $D_{2}$, that is $p>C\left(D_{2}\right)$ with $C\left(D_{2}\right)$ effectively computable, then apart from the pairs $\left(p, D_{2}\right)$ belonging to (6) we have $N\left(1,1, D_{2}, p\right) \leq 1$. As a consequence of a more general result, Le [49] proved that there exists an absolute constant $C_{1}$ such that $N\left(1,1, D_{2}, p\right) \leq 1$ if $\max \left\{D_{2}, p\right\}>C_{1}$, unless $\left(p, D_{2}\right)$ is given by (6). It was already noted by Beukers [17] that distinct solutions in positive integers $x$ and $n$ to (5) correspond to integers $m \geq 2$ for which

$$
\frac{\lambda^{m}-\lambda_{c}^{m}}{\lambda-\lambda_{c}}= \pm 1
$$

where $\lambda$ is an algebraic integer in $\mathbb{Q}\left(\sqrt{-D_{2}}\right)$ and $\lambda_{c}$ denotes the complex conjugate of $\lambda$. By applying the powerful result of Bilu, Hanrot and Voutier [19] on primitive divisors of Lucas and Lehmer sequences, Bugeaud and Shorey [25] improved the above mentioned results of Bender and Herzberg and Le. They proved, among others, that equation (5) has at most one solution, unless $\left(p, D_{2}\right)$ is given by (6) (i.e. $N\left(1,1, D_{2}, p\right) \leq 1$ holds if and only if $\left(p, D_{2}\right)$ is not of the form (6)).

In a series of papers Le [46]-[52] investigated equation (4) with

$$
D_{1} \geq 1, D_{2} \geq 1, a \in\{1,2,4\}, \operatorname{gcd}\left(b, D_{1} D_{2}\right)=1 \text { and } a=4 \text { if } b=2 .
$$

He then obtained many results on equation (4) including an uniform upper bound for the number of solutions $N\left(a, D_{1}, D_{2}, p\right)$ of (4). More precisely, he proved that for equation (4) satisfying assumptions (7) with $b=p$ a prime one has

$$
N\left(a, D_{1}, D_{2}, p\right) \leq 2
$$

except for $N(4,1,7,2)=5$ and $N(4,3,5,2)=N(4,1,11,3)=N(4,1,19,5)$

$=3($ see $[51],[52])$.

We mention that the above theorem is sharp in the sense that there are infinite families of $\left(D_{1}, D_{2}, p\right)$ with $N\left(1,1, D_{2}, p\right)=2$ (see (6)). By using the above mentioned result of Bilu, Hanrot and Voutier on primitive divisors of Luca and Lehmer sequences, Bugeaud and Shorey [25] considerably improved this result by means of the exact determination of all triples $\left(D_{1}, D_{2}, p\right)$ for which equation (4) under assumptions (7) has more than one solution. Namely, they proved that if one considers equation (4) satisfying assumptions (7) with $b=p$ a prime then we have

$$
N\left(a, D_{1}, D_{2}, p\right) \leq 1,
$$


except for $N(4,13,3,2)=N(2,7,11,3)=N(4,7,1,2)=N(2,1,1,5)=$ $N(2,1,1,13)=N(4,1,3,7)=N(1,6,1,7)=2, N(1,2,1,3)=3$, and when $\left(D_{1}, D_{2}, p\right)$ belongs to one of three explicitly given infinite families (see the terminology of [25]). In the latter case, we always have $N\left(a, D_{1}, D_{2}, p\right)=2$, except for $N(4,1,7,2)=5$ and $N(4,3,5,2)=N(4,1,11,3)=N(4,1,19,5)=$ 3 . Note, that the cases $N(1,2,1,3)=3$ and $N(1,6,1,7)=2$ were proved by Leu and Li [58] and Mollin [67], respectively.

By applying the theory of binary quadratic forms Saradha and Srinivasan [78] gave a necessary condition under which equation (4) has no solution. Using this result they solved completely several equations of the form (4) with $D_{1}=1$ and $b=p$, a prime (see e.g. Theorems 1.3-1.6 of [78]). Equation (4) was also considered in the case when $b$ is a positive composite integer. For example, Bugeaud and Shorey [25] solved completely equation (4) with $\left(a, D_{1}, D_{2}, b\right) \in\{(1,1,19,55),(1,1,341,377)\}$.

\subsection{Upper bounds for $N\left(a, D_{1}, D_{2}, b\right)$ with $D_{2}<0$}

In [17] Beukers dealt also with the case $D_{2}<0$ and showed that if $a=1, D_{1}=$ $1, b=2$ then equation (4) has at most four solutions for every $D_{2}<0$, that is $N\left(1,1, D_{2}, 2\right) \leq 4$. This result is sharp since there exist infinitely many values of $D_{2}$ for which (4) has exactly four solutions with $a=1, D_{1}=1, b=2$. Namely, if $D_{2}=-\left(2^{2 k}-3 \cdot 2^{k+1}+1\right), k \geq 3$ then

$$
(x, n)=\left(2^{k}-3,3\right),\left(2^{k}-1, k+2\right),\left(2^{k}+1, k+3\right),\left(3 \cdot 2^{k}-1,2 k+3\right)
$$

are solutions of the equation (4) with $a=1, D_{1}=1, b=2$. Le [45] improved this result by proving among others that if $D_{2} \neq-\left(2^{2 k}-3 \cdot 2^{k+1}+1\right)$ with $k \geq 3$, then there are at most three solutions to (4) with $a=1, D_{1}=1, b=2$. In [18] Beukers extended his investigations concerning equation (4) wit $b=p$ an odd prime. He showed that if $a=D_{1}=1, D_{2}<0$ is fixed and $p$ an odd prime not dividing $D_{2}$ then equation (4) has at most four solutions (i.e. $\left.N\left(1,1, D_{2}, p\right) \leq 4\right)$. Further, he gave a family of such equations having three solutions. Namely, let $m \geq 1, l \geq 1, \varepsilon \in\{ \pm 1\}$ and suppose that $p$ is an odd prime of the form $p=4 m^{2}+\varepsilon$ and let $D_{2}=\left(\left(p^{l}-\varepsilon\right) / 4 m\right)^{2}-p^{l}$. Then equation (4) with $a=1, D_{1}=1, b=p$ has the following solutions

$$
(x, n)=\left(\frac{p^{l}-\varepsilon}{4 m} \cdot 2 m, 1\right),\left(\frac{p^{l}-\varepsilon}{4 m}, l\right),\left(2 m p^{l}+\varepsilon \frac{p^{l}-\varepsilon}{4 m}, 2 l+1\right) .
$$

Here we mention a result of Yuan [95], who proved that $N\left(1,1, D_{2}, p\right) \leq 3$ if $\max \left(p, D_{2}\right)>10^{32}$ and $\left(p, D_{2}\right) \neq\left(4 m^{2}+\varepsilon,\left(\left(p^{l}-\varepsilon\right) / 4 m\right)^{2}-p^{l}\right)$. By combining the hypergeometric method with lower bounds for linear forms in logarithms 
of algebraic numbers Bauer and Bennett [10] improved the above result of Beukers and Yuan by proving that $N\left(1,1, D_{2}, p\right) \leq 3$ holds for (4), where $D_{2}<0$ is a given integer and $p$ is in odd prime not dividing $D_{2}$.

For further generalizations and extensions of equations of Ramanujan- Nagell type (4) the interested reader may consult some results of de Weger [92]. By applying the theory on linear forms in logarithms of algebraic numbers, the basis reduction algorithm of Lenstra, Lenstra and Lovász [57], and the Fincke-Pohst algorithm (see [39]) for finding short lattice vectors, de Weger [92] extended the theory into two more directions. In the first place he studied for fixed integer $k$ and primes $p_{1}, \ldots, p_{s}$ the generalized Ramanujan-Nagell equation

$$
x^{2}+k=p_{1}^{z_{1}} \cdots p_{s}^{z_{s}}
$$

in unknown integers $x, z_{1}, \ldots, z_{s}$ with $x \geq 1, z_{i} \geq 0(1 \leq i \leq k)$. As an illustration of his method he computed all 16 nonnegative numbers $x$ such that $x^{2}+7$ has no prime divisors larger than 20 explicitly, the largest being $y=273$. Secondly, he considered the equation

$$
x+z=y^{2}
$$

in integers $x, y, z$ with $x>z, y>0, x$ and $|z|$ composed of fixed primes. As an illustration he computed all 388 solutions where $x$ and $|z|$ are composed of the primes $2,3,5$ and 7 , the largest solution being $199290375-686=14117^{2}$. For further developments into this direction see e.g. Smart [83] and Wildanger [93].

\section{Generalized Lebesque-Nagell equations}

There are many results concerning the equation

$$
x^{2}+D=y^{n},
$$

where $D>0$ is a given integer and $x, y, n$ are positive integer unknowns with $n \geq 3$. Results obtained for general superelliptic equations clearly provide effective finiteness results for this equation, too (see for example [82], [80], [14] and the references given there).

\subsection{The equation $x^{2}+D=y^{n}$ with fixed $D$}

The first result concerning the above equation was due to V. A. Lebesgue [56] who proved that there are no solutions for $D=1$. Nagell [69],[71] solved it for $D=3,4$ and 5 . It is for this reason that equation (8) is called the 
Lebesgue-Nagell equation. Ljunggren [59] solved completely (8) for $D=2$ (see also [60]). In his elegant paper [35], Cohn gave a fine summary of the earlier results on equation (8). Further, he developed a method by which he found all solutions of the above equation for 77 positive values of $D \leq 100$ (see also [36]). For $D=74$ and $D=86$, equation (8) was solved by Mignotte and de Weger [66]. By using the theory of Galois representations and modular forms Bennett and Skinner [13] solved (8) for $D=55$ and $D=95$. On combining the theory of linear forms in logarithms with Bennett and Skinner's method and with several additional ideas, Bugeaud, Mignotte and Siksek [23] gave all the solutions of (8) for the remaining 19 values of $D \leq 100$.

\subsection{The equation $x^{2}+D=y^{n}$ with $D$ in some infinite set}

Let $S=\left\{p_{1}, \ldots, p_{s}\right\}$ denote a set of distinct primes and $\mathbf{S}$ the set of nonzero integers composed only of primes from $S$. Put $P:=\max \left\{p_{1}, \ldots, p_{s}\right\}$ and denote by $Q$ the product of the primes of $S$. In recent years, equation (8) has been considered also in the more general case when $D$ is no longer fixed but $D \in \mathbf{S}$ with $D>0$. It follows from Theorem 2 of Shorey, van der Poorten, Tijdeman and Schinzel [81] that in (8) $n$ can be bounded from above by an effectively computable constant depending only on $P$ and $s$. Győry, Pink and Pintér [42] derived an effective upper bound for $n$ which depends only on $Q$. Cohn [34] showed that if $D=2^{2 k+1}$ then equation (8) has solutions only when $n=3$ and in this case there are three families of solutions. The case $D=2^{2 k}$ was considered by Arif and Abu Muriefah [5]. They conjectured that the only solutions are given by $(x, y)=\left(2^{k}, 2^{2 k+1}\right)$ and $(x, y)=\left(11 \cdot 2^{k-1}, 5 \cdot 2^{2(k-1) / 3}\right)$, with the latter solution existing only when $(k, n)=(3 M+1,3)$ for some integer $M \geq 0$. Partial results towards this conjecture were obtained in [5] and [33] and it was finally proved by Arif and Abu Muriefah [8]. For an other result concerning the case $D=2^{a}$ we refer to [53]. Arif and Abu Muriefah [6] proved that if $D=3^{2 k+1}$ then (8) has exactly one infinite family of solutions. The case $D=3^{2 k}$ has been solved by Luca [61] under the additional hypothesis that $x$ and $y$ are coprime. This was extended by Tao [87] to arbitrary positive integers $x, y$ (and $D=3^{2 k}$ ). In [62] Luca solved completely equation (8) if $D=2^{a} 3^{b}$ and $\operatorname{gcd}(x, y)=1$. Abu Muriefah [1] established that equation (8) with $D=5^{2 k}$ may have a solution only if 5 divides $x$ and $p$ does not divide $k$ for any odd prime $p$ dividing $n$. For related results concerning the case $D=5^{m}$ one may consult the paper of Tao [88]. The case $D=2^{a} 3^{b} 5^{c} 7^{d}$

with $\operatorname{gcd}(x, y)=1$, where $a, b, c, d$ are non-negative integers was studied by Pink [74] and for $a \geq 1$ and $\operatorname{gcd}(x, y)=1$ all the solutions of (8) were listed. The complete solution of (8) in the cases when $D=7^{2 k}$ and $D=2^{a} 5^{b}$ were given by Luca and Togbe [64], [65]. In [68] Abu Muriefah, Luca and Togbe determined all solutions of (8) with $D=5^{a} 13^{b}$ and $\operatorname{gcd}(x, y)=1$. Further, in 
this paper the authors gave a summary on the methods dealing with equation of type (8). In the case $D=2^{a} 5^{b} 13^{c}$ equation (8) was handled by Goins, Luca and Togbe [41].

Let $p \geq 5$ be an odd prime with $p \neq \equiv 7(\bmod 8)$. Arif and Abu Muriefah [9] determined all solutions of equation (8) with $D=p^{2 k+1}$, where $\operatorname{gcd}\left(n, 3 h_{0}\right)=1$ and $n \geq 3$. Here $h_{0}$ denotes the class number of the field $\mathbb{Q}(\sqrt{-p})$. In [7] they also obtained partial results if $D=p^{2 k}$, where $p$ is an odd prime. In the particular case when $\operatorname{gcd}(x, y)=1, D=p^{2}, p$ prime with $3 \leq p<100$ Le [54] gave all the solutions of equation (8). The case $D=p^{2 k}$ with $2 \leq p<100$ prime and $\operatorname{gcd}(x, y)=1$ was solved by Bérczes and Pink [15]. If in $(8) D=a^{2}$ with $3 \leq a \leq 501$ and $a$ odd Tengely [89] solved completely equation (8) under the assumption $(x, y) \in \mathbb{N}^{2}, \operatorname{gcd}(x, y)=1$. The equation $A^{4}+B^{2}=C^{n}$ for $A B \neq 0$ and $n \geq 4$ was completely solved by Bennett, Ellenberg and Ng [12] by means of the modular method (see also Ellenberg [38]). In [77] Saradha and Srinivasan discussed equation (8) for $D=p_{1}^{\alpha_{1}} \cdots p_{r}^{\alpha_{r}}=D_{s} D_{t}^{2}$, where $D_{s}$ is the square-free part of $D$ and $\alpha_{1}, \ldots \alpha_{r}$ are nonnegative integer unknowns. They obtained several interesting results concerning the case $D_{s} \leq 10000$. Further, for the case $D=p^{l}, p \in\{11,19,43,67,163\}$, it was proved in the same paper [77] that equation (8) may have a solution if $l=3^{\beta} 5^{\gamma}$.

Le and Zhu [55] solved completely equation (8) with $D=p^{l}$ with $p \in$ $\{11,19,43,67,163\}$, where the class number $h(-p)=1$. Here $h(-p)$ denotes the class number of the imaginary quadratic field $\mathbb{Q}(\sqrt{-p})$. In [96] Zhu discussed equation (8) with $D=p^{a}, p$ a prime and $n=3$. For an other result with $D=p^{a}$ we refer to Bugeaud [22]. Bérczes and Pink [16] extending the above result of Saradha and Srinivasan and Le and Zhu solved completely equation (8) for $D=d^{2 l+1}$ in the case $h(-d) \in\{2,3\}$, where $d>0$ is a square-free integer and $h(-d)$ is the class number of the imaginary quadratic field $\mathbb{Q}(\sqrt{-d})$. Cenberci and Senay [30] considered equation (8) with $D=q^{m}, y=p$, where $p$ and $q$ are odd primes satisfying $q^{2}+1=2 p^{2}$ and other additional conditions. Demirpolat, Cenberci and Senay [37] established that equation (8) with $D=11^{2 k+1}$ has exactly only one family of solution, when $n$ is an odd integer. Cangül, Soydan and Simsek [29] solved completely equation (8) with $D=11^{2 k}$. Cangül, Demirci, Luca, Pintér and Soydan [27] found all solutions of (8) with $D=2^{a} 11^{b}$ and $\operatorname{gcd}(x, y)=1$. The case $D=2^{a} 3^{b} 11^{c}$ has been considered by Cangül, Demirci, Inam, Luca and Soydan [28], where all the solutions of $(8)$ were given with $\operatorname{gcd}(x, y)=1$. Except for the case when $x a b$ is odd, the complete solution of (8) with $D=5^{a} 11^{b}$ and $\operatorname{gcd}(x, y)=1$ has been obtained by Cangül, Demirci, Soydan and Tzanakis [26]. Pink and Rábai [75] gave all solutions of equation $(8)$ if $D=5^{a} 17^{b}$ and $\operatorname{gcd}(x, y)=1$. Godinho, Marques and Togbe [40] solved completely (8) the case $D=2^{a} 5^{b} 17^{c}$ under the assumption $\operatorname{gcd}(x, y)=1$. Soydan [84] gave all the solutions of the Diophan- 
tine equation (8) with $D=7^{a} 11^{b}$ for the nonnegative integers $a, b, x, y, n \geq 3$, where $x$ and $y$ are coprime, except when $a, x$ is odd and $b$ is even. Cenberci and Peker [31] treated the case $D=19^{a}$, while Soydan, Ulas and Zhu [85] solved completely (8) with $D=2^{a} 19^{b}$ and $\operatorname{gcd}(x, y)=1$. Xiaowei [94] gave a classification of solutions of equation (8) with $D=p^{2 k}$ and $\operatorname{gcd}(x, y)=1$.

There are many papers in the literature concerning various extensions and generalizations of equations of type (8). Namely, several authors considered the equation

$$
x^{2}+D=b y^{n},
$$

where $b$ is a nonnegative integer which is not necessarily 1 . To give an exhaustive survey on results in this direction is beyond the scope of this paper. Hence we only mention a few papers dealing with equations of type (9). The interested reader may consult these papers an the references therein.

In connection with equation (9) we only mention some results of Bugeaud and Shorey [25], where among other important resuts, they solved completely (9) with $(D, b)=(7,4)$. Further, in the same paper they determined all the solutions of $(9)$ when $D$ is square-free, $D \not \equiv 7(\bmod 8)$ and $h(-4 D)$ equals 1 or a power of 2 , where $h(-4 D)$ equals the class number of the unique quadratic order of discriminant $-4 D$. Tengely [89] solved completely equation (9) with $(D, b)=\left(a^{2}, 2\right)$ odd prime $n$ for all $a \in\{3,4, \ldots, 501\}$. In [90] Tengely proved that equation (9) with $(D, b)=\left(q^{2 m}, 2\right)$, where $q$ and $n>3$ are odd primes has finitely many solutions $(x, y, m, n, q)$ under the assumption that $y$ is not the sum of two consecutive intgers. Further, he gave all the solutions of (9) in the case $(D, b)=\left(3^{2 m}, 2\right)$. Abu Muriefah, Luca, Siksek and Tengely [2] considered equation (9) with $b=2$ and $D \equiv 1(\bmod 4)$ and gave some sharp conditions for $n$ to be satisfied, in order to have a solution. Luca, Tengely and Togbe [63] solved completely $(9)$ with $b=4$ and $D \equiv 3(\bmod 4), 1 \leq D \leq 100$. For other related results concerning equations (8) and (9) we mention some excellent surveys of Abu Muriefah and Bugeaud [24] and Saradha and Srinivasan [79]. For recent surveys we refer to $\mathrm{Hu}$ and Le [44] and Virgolici [91], respectively.

\section{The "classical" method and a new result}

In this section we would like to present the method which is most important for the proof of most results claiming complete solution of Lebesgue-Nagell type equations. The key ingredient of this method is the powerful result of Bilu, Hanrot and Voutier [19] concerning primitive prime prime divisors of Lucas sequences. To illustrate the method we solve completely the equation

$$
x^{2}+11^{a} 17^{b}=y^{n}
$$


in nonnegative integer unknowns $(x, y, a, b, n)$ with

$$
x \geq 1, y \geq 2, a \geq 0, b \geq 0, n \geq 3 \text { and } \operatorname{gcd}(x, y)=1 .
$$

Theorem 3.1. Consider equation (10) satisfying (11). Then all solutions of (10) are

$$
(x, y, a, b, n) \in\{(4,3,1,0,3),(58,15,1,0,3),(2,5,2,0,3),(9324,443,3,0,3),
$$$$
(14,15,1,2,3),(7670,389,2,2,3),(670,111,1,4,3),(8,3,0,1,4)\} .
$$

Proof. We note that since $n \geq 3$, it follows that $n$ is either a multiple of 4 , or $n$ is a multiple of an odd prime $p$. Furthermore, if $d \mid n$ is such that $d \in\{4, p\}$ with $p$ an odd prime and $(x, y, a, b, n)$ is a solution of our equation (10), then $\left(x, y^{n / d}, a, b, d\right)$ is also a solution of our equation (10) satisfying the same restrictions. Thus, we may replace $n$ by $d$ and $y$ by $y^{n} / d$, and from now on assume that $n \in\{3,4\}$ or $n \geq 5$ a prime. Hence, in what follows we will distinguish between the cases $n \in\{3,4\}$ and $n \geq 5$ a prime.

The cases $n \in\{3,4\}$.

In these cases we apply the following approach: we transform equation (10) into several elliptic equations written in cubic and quartic models, respectively, for which we need to determine all their $\{11,17\}$-integral points.

For $n=3$ we write $a$ and $b$ in the form $a=6 a_{1}+a_{2}, b=6 b_{1}+b_{2}$, where $a_{1}, b_{1}, a_{2}, b_{2}$ are nonnegative integers with $a_{2}, b_{2} \in\{0,1,2,3,4,5\}$. Hence equation (10) takes the form

$$
X^{2}=Y^{3}-11^{a_{2}} 17^{b_{2}}, \quad a_{2}, b_{2} \in\{0,1,2,3,4,5\},
$$

where

$$
X=\frac{x}{11^{3 a_{1}} 17^{3 b_{1}}}, Y=\frac{y}{11^{2 a_{1}} 17^{2 b_{1}}} .
$$

Now, we use an appropriate program package (e.g. MAGMA [20]) to determine the $S$-integral points on the elliptic curves occurring in (12), where in our case $S=\{11,17\}$. The solutions to (12) satisfying $X>0$ and $Y>0$ are

$$
\begin{gathered}
\left(X, Y, a_{2}, b_{2}\right) \in\{(4,3,1,0),(58,15,1,0),(2,5,2,0), \\
(9324,443,3,0),(68,17,0,2),(14,15,1,2),(7670,389,2,2), \\
(34848,1067,4,2),(5060,297,2,3),(670,111,1,4)\} .
\end{gathered}
$$

Then, by using substitution (13) we infer that the solutions of our original equation (10) satisfying (11) with $n=3$ are

$$
(x, y, a, b, n)=\{(4,3,1,0,3),(58,15,1,0,3),(2,5,2,0,3),(9324,443,3,0,3),
$$


$(14,15,1,2,3),(7670,389,2,2,3),(670,111,1,4,3)\}$.

Next, we consider equation (10) satisfying (11) with $n=4$. We apply a similar argument as above. Namely, if $n=4$ we reduce our equation (10) to several elliptic curves of Ljunggren-type. For this, consider (10) and write $a$ and $b$ now in the form $a=4 a_{1}+a_{2}, b=4 b_{1}+b_{2}$, where $a_{1}, b_{1}, a_{2}, b_{2}$ are nonnegative integers with $a_{2}, b_{2} \in\{0,1,2,3\}$. Hence equation (10) takes the form

$$
X^{2}=Y^{4}-11^{a_{2}} 17^{b_{2}}, \quad a_{2}, b_{2} \in\{0,1,2,3\}
$$

where

$$
X=\frac{x}{11^{2 a_{1}} 17^{2 b_{1}}}, Y=\frac{y}{11^{a_{1}} 17^{b_{1}}} .
$$

We use MAGMA again to find all S-integral points on the Ljunggren-type curve (14) and to conclude that the only solution of (14) with $X>0$ and $Y>0$ is $\left(X, Y, a_{2}, b_{2}\right)=(8,3,0,1)$. This implies, via the transformation (15), that our original equation (10) has only one solution satisfying (11) with $n=4$. Namely, we get $(x, y, a, b, n)=(8,3,0,1,4)$.

The case $n \geq 5$ a prime.

In this case the Primitive Divisor Theorem of Bilu, Hanrot and Voutier [19] can be applied very efficiently.

Recall that a Lucas-pair is a pair $(\alpha, \beta)$ of algebraic integers such that $\alpha+\beta$ and $\alpha \beta$ are non-zero coprime rational integers and $\alpha / \beta$ is not a root of unity. Given a Lucas-pair $(\alpha, \beta)$ one defines the corresponding Lucas sequence by

$$
L_{n}=\frac{\alpha^{n}-\beta^{n}}{\alpha-\beta},(n=0,1,2 \ldots) .
$$

A prime number $p$ is called a primitive divisor of $L_{n}$ if $p$ divides $L_{n}$ but does not divide $(\alpha-\beta)^{2} L_{1} \cdots L_{n-1}$.

Lemma 3.2 (Primitive Divisor Theorem). Let $L_{n}=L_{n}(\alpha, \beta)$ be a Lucas sequence. If $n \geq 5$ is a prime then $L_{n}$ has a primitive prime divisor except for finitely many pairs $(\alpha, \beta)$ which are explicitly determined in Table 1 of [19].

We are now in position to finish the proof of Theorem 3.1. We write equation (10) in the form

$$
x^{2}+d c^{2}=y^{n},
$$

where $d c^{2}=11^{a} 17^{b}$ and $d>0$ is the square-free part of $11^{a} 17^{b}$, that is $d \in\{1,11,17,187\}$ according to the parities of the exponents $a$ and $b$. By factorizing the above equation in the imaginary quadratic field $\mathbb{K}=\mathbb{Q}(\sqrt{-\bar{d}})$ we get

$$
(x+c \sqrt{-d})(x-c \sqrt{-d})=y^{n} .
$$


By reducing equation (10) modulo 8 we easily see that $x$ is even and $y$ is odd. Since $\operatorname{gcd}(x, y)=1$, a standard argument shows that the ideals $(x+c \sqrt{-d})$ and $(x-c \sqrt{-d})$ are coprime in $\mathbb{K}$. The class number $h(-d)$ of $\mathbb{K}=\mathbb{Q}(\sqrt{-d})$ belongs to $\{1,1,4,2\}$ according to $d \in\{1,11,17,187\}$, respectively. Since $n \geq 5$ is prime we obtain that $\operatorname{gcd}(h(-d), n)=1$ and so $(16)$ implies that

$$
x+c \sqrt{-d}=\varepsilon \delta^{n},
$$

where $\delta$ is an algebraic integer in $\mathbb{K}$ and $\varepsilon$ is a unit in $\mathbb{K}$. The group of units of $\mathbb{K}$ is $\mathcal{U}_{\mathbb{K}}=\{ \pm 1, \pm i\}$ if $d=1$ and $\mathcal{U}_{\mathbb{K}}=\{ \pm 1\}$ if $d \in\{11,17,187\}$. Thus, since $n \geq 5$ is prime, the unit factor $\varepsilon$ occurring in (17) can be absorbed into $\delta^{n}$. More precisely, we may write

$$
x+c \sqrt{-d}=\gamma^{n}
$$

for some algebraic integer $\gamma$. Furthermore, $\{1, \sqrt{-d}\}$ is an integral basis for $O_{\mathbb{K}}$ if $d \in\{1,17\}$, while if $d \in\{11,187\}$ then $\left\{1, \frac{1+\sqrt{-d}}{2}\right\}$ is an integral basis for $O_{\mathbb{K}}$. Hence we may write $\gamma$ occurring in (18) in the form

$$
\gamma=u+v \sqrt{-d}
$$

where either both $u$ and $v$ are integers, or both $2 u$ and $2 v$ are odd integers, the last case occurring when $d=11$ or $d=187$. By conjugating relation (18) and using (19) we get

$$
L_{n}=\frac{\gamma^{n}-\bar{\gamma}^{n}}{\gamma-\bar{\gamma}}=\frac{(u+v \sqrt{-d})^{n}-(u-v \sqrt{-d})^{n}}{2 v \sqrt{-d}}=\frac{c}{v}
$$

We easily see that the sequence $L_{n}$ occurring in (20) is a Lucas sequence with the choice $\alpha=u+v \sqrt{-d}$ and $\beta=u-v \sqrt{-d}$. Further, obviously $v \mid c$ holds and the prime factors of $c$ belong to $\{11,17\}$. By Lemma 3.2 we conclude that for $n \geq 5$ prime the Lucas sequence $L_{n}$ always has a primitive prime divisor except for finitely many exceptions, which are explicitely given in Table 1 of [19]. Consider first the case when $L_{n}$ does not have a primitive prime divisor. Now, a quick look at Table 1 of [19] reveals that we necessarily have

$$
n=5, \gamma=\frac{1+\sqrt{-11}}{2} \text { and } \bar{\gamma}=\frac{1-\sqrt{-11}}{2}
$$

which leads to $y=\gamma \bar{\gamma}=3$. Hence using (10) we get that $3^{5}=x^{2}+11^{a} 17^{b}$, implying that our equation (10) does not have a solution with $n \geq 5$ in this case. 
Next consider the case when $L_{n}$ appearing in (20) has a primitive prime divisor $p$. It is well known, that if such a $p$ exists then it satisfies $p \equiv \pm 1$ $(\bmod n)$, where the sign coincides with

$$
\left(\frac{(\gamma-\bar{\gamma})^{2}}{p}\right)=\left(\frac{-4 d v^{2}}{p}\right)=\left(\frac{-d}{p}\right) .
$$

Here $(\vdots)$ stands for the Legendre symbol. Clearly $p \in\{11,17\}$ and hence

$$
n \mid 11 \pm 1 \text { or } n \mid 17 \pm 1 .
$$

Since $n \geq 5$ is prime (23) implies that the only possibility for $p$ to be a primitive prime divisor of $L_{n}$ is

$$
p=11
$$

occurring only if $n=5$ and $d=17$. To finish the proof we have to solve our original equation (10) with $d=17$ and $n=5$. In this case equation (10) takes the form

$$
x^{2}+17 c^{2}=y^{5},
$$

where $c=11^{a / 2} 17^{b-1 / 2}$ with $a$ even and $b$ odd. By using (20) with $n=5$ and $d=17$ we get

$$
L_{5}=c / v,
$$

which by $L_{5}=5 u^{4}-170 u^{2} v^{2}+289 v^{4}$ and $c=11^{a / 2} 17^{b-1 / 2}$ can be rewritten as

$$
v\left(5 u^{4}-170 u^{2} v^{2}+289 v^{4}\right)=11^{a / 2} 17^{b-1 / 2} .
$$

Since $u$ and $v$ are coprime, we have the possibilities

$$
v= \pm 11^{a / 2} 17^{b-1 / 2}, v= \pm 11^{a / 2}, v= \pm 17^{b-1 / 2}, v= \pm 1 .
$$

By using (24) and (25) we easily see that the first two cases lead again to the conclusion that $L_{5}$ has no primitive prime divisor, which is impossible. So we look at the last two possibilities.

If $v= \pm 17^{b-1 / 2}$ we obtain by (26) that

$$
5 u^{4}-170 u^{2} v^{2}+289 v^{4}= \pm 11^{a / 2} .
$$

On dividing both sides of (28) by $v^{4}$ we obtain the Ljunggren-type curves

$$
5 T^{4}-170 T^{2}+289= \pm V W^{2},
$$

where $V \in\{ \pm 1, \pm 11\}$ and $T=u / v, W=11^{a / 4} / v^{2}$ if $a / 2$ is even and $T=$ $u / v, W=11^{a-2 / 4}$ if $a / 2$ is odd. Since $v= \pm 17^{b-1 / 2}$ we observe that we have to give all the 17-integral points on the curves defined by (29). By using 
MAGMA we get that the only $\{17\}$-integral point on the curves in (29) is $(T, W)=(0, \pm 17)$ occurring if $V=1$. Since $T=u / v$ this leads to $u=x=0$ which is a contradiction in view of (11).

Finally, if $v= \pm 1$ we obtain by (26) that

$$
5 u^{4}-170 u^{2} v^{2}+289 v^{4}= \pm 11^{a / 2} 17^{b-1 / 2} .
$$

We follow the above approach to get the curves

$$
5 T^{4}-170 T^{2}+289= \pm V W^{2},
$$

where $V \in\{1,11,17,187\}$. By using MAGMA again, we obtain that the only $\{17\}$-integral point on the curves in $(30)$ is $(T, W)=(0, \pm 17)$, occurring if $V=1$, whence we arrive, as above, to $x=0$. Hence we may conclude that our equation (10) has no solutions satisfying (11) with $n \geq 5$ prime.

Remark. In the case $n \in\{3,4\}$ we have to search the $S$-integral points on some elliptic curves written in cubic and quartic models. The resolution of such equations can be accomplished by the method of Pethö et al. [73] and Stroeker and Tzanakis [86]. The powerful theorem of Bilu, Hanrot and Voutier applied for the case $n \geq 5$ works also for other values of $D=p_{1}^{a_{1}} \cdots p_{k}^{a_{k}}$, where $p_{1}, \ldots, p_{k}$ are fixed primes provided that some conditions are satisfied (see for example [24], [68]). Moreover, in some cases we have to solve an equation of the form $F(U, V)=W$, where both $V$ and $W$ are $S$-units for $S=\left\{p_{1}, \ldots p_{k}\right\}$ and $F$ is a homogenous polynomial of degree $(n-1) / 2$. Thus, the last necessary condition is that we can find all the solutions of these equations which in several cases lead to curves of genus 2 .

Acknowledgement. The publication of this article is supported by the grant of CNCS-UEFISCDI (Romanian National Authority for Scientic Research) PN-II-ID-WE-2012-4-161 of the workshop "Workshop on Algebraic and Analytic Number Theory and their Applications", Constanta, May 23-24, 2013.

\section{References}

[1] F.S. Abu Muriefah, On the Diophantine equation $x^{2}+5^{2 k}=y^{n}$, Demonstratio Mathematica 319/2 (2006), 285-289.

[2] F.S. Abu Muriefah, F. Luca, S. Siksek, and Sz. Tengely, On the Diophantine equation $x^{2}+C=2 y^{n}$, Int. J. Number Theory $\mathbf{5}$ (2009), 1117-1128. 
[3] R. Apéry, Sur une equation diophantienne (French), C. R. Acad. Sci. Paris 251 1960, 1263-1264.

[4] R. Apéry, Sur une equation diophantienne (French), C. R. Acad. Sci. Paris 251 1960, 1451-1452.

[5] S.A. Arif and F.S.A. Muriefah, On the Diophantine equation $x^{2}+$ $2^{k}=y^{n}$, Internat. J. Math. Math. Sci. 20 (1997), 299-304.

[6] S.A. Arif and F.S.A. Muriefah, The Diophantine equation $x^{2}+$ $3^{m}=y^{n}$, Internat. J. Math. Math. Sci. 21 (1998), 619-620.

[7] S.A. Arif and F.S.A. Muriefah, The Diophantine equation $x^{2}+$ $q^{2 k}=y^{n}$, Arab. J. Sci. Sect. A Sci. 26 (2001), 53-62.

[8] S.A. Arif and F.S.A. Muriefah, On the Diophantine equation $x^{2}+$ $2^{k}=y^{n}$ II, Arab J. Math. Sci. 7 (2001), 67-71.

[9] S.A. Arif and F.S.A. Muriefah, On the Diophantine equation $x^{2}+$ $q^{2 k+1}=y^{n}$, J. Number Theory 95 (2002), 95-100.

[10] M. Bauer and M. A. Bennett, Applications of the hypergeometric method to the generalized Ramanujan-Nagell equation, Ramanujan J. 6 (2002), 209-270.

[11] E. A. Bender, N. P. Herzberg, Some Diophantine equations related to the quadratic form $a x^{2}+b y^{2}$, in: Studies in Algebra and Number Theory, Academic Press, New York 1979, 219-272.

[12] M.A. Bennett, J.S. Ellenberg and N. Ng, The Diophantine equation $A^{4}+2^{d} B^{2}=C^{n}$ Inter. J. Number Theory 6 (2010), 1-27.

[13] M.A. Bennett and C.M. Skinner, Ternary diophantine equations via Galois representations and modular forms, Canad. J. Math. 56/1, (2004), 23-54.

[14] A. Bérczes, B. Brindza and L. Hajdu, On power values of polynomials, Publ. Math. Debrecen, 53, (1998), 375-381.

[15] A. Bérczes and I. Pink, On the diophantine equation $x^{2}+p^{2 k}=y^{n}$, Archiv der Mathematik, 91 (2008), 505-517.

[16] A. Bérczes and I. Pink, On the Diophantine equation $x^{2}+d^{2 l+1}=$ $y^{n}$, Glasg. Math. Journal, 54 (2012), 415-428.

[17] F. Beukers, On the generalized Ramanujan-Nagell equation I, Actha Arith. 38 (1980/1981), 389-410. 
[18] F. Beukers, On the generalized Ramanujan-Nagell equation II, Actha Arith. 39 (1981), 113-123.

[19] Y. Bilu, G. Hanrot and P.M. Voutier, Existence of primitive divisors of Lucas and Lehmer numbers. With an appendix by $M$. Mignotte., J. Reine Angew. Math. 539 (2001), 75-122.

[20] W. Bosma, J. Cannon and C. Playoust, The Magma algebra system. I. The user language, J. Symbolic Comput. 24 (1997), 235265.

[21] J. Browkin and A. Schinzel, On the equation $2^{n}-D=y^{2}$, Bull. Acad. Polon. Sci. Sr. Sci. Math. Astronom. Phys. 8 (1960), 311318.

[22] Y. Bugeaud On the diophantine equation $x^{2}-p^{m}= \pm y^{n}$, Acta Arith. 80, (1997), 213-223.

[23] Y. Bugeaud, M. Mignotte and S. Siksek, Classical and modular approaches to exponential and diophantine equations II. The Lebesque-Nagell equation, Compos. Math. 142/1 (2006), 31-62.

[24] Y. Bugeaud and F.S. Abu Muriefah, The Diophantine equation $x^{2}+c=y^{n}$ : a brief overview, Revista Colombiana de Matematicas, 40, (2006), 31-37.

[25] Y. Bugeaud and T.N. Shorey, On the number of solutions of the generalized Ramanujan-Nagell equation, J. reine angew. Math. 539 (2001), 55-74.

[26] I.N. Cangül, M. Demirci, G. Soydan, N. Tzanakis, On the Diophantine equation $x^{2}+5^{a} 11^{b}=y^{n}$, Funct. Approx. Comment. Math. 43 (2010), 209-225.

[27] I. N. Cangül, M. Demirci, F. Luca, Á. Pintér and G. Soydan, On the Diophantine equation $x^{2}+2^{a} 11^{b}=y^{n}$, Fibonacci Quart. 48 (2010), 39-46.

[28] I. N. Cangül, M. Demirci, I. Inam, F. Luca and G. Soydan, $O N$ THE DIOPHANTINE EQUATION $x^{2}+2^{a} 3^{b} 11^{c}=y^{n}$, Math. Slovaca 63 (2013), 647-659.

[29] I. N. Cangül, G. Soydan and Y. Simsek, A p-adic Look at the Diophantine Equation $x^{2}+11^{2 k}=y^{n}$, AIP Conf. Proc. 1168 (2011), 275-277. 
[30] S. Cerberci and H. Senay, The Diophantine Equation $x^{2}+q^{m}=p^{n}$, Int. J. Contemp. Math. Sciences 24 (2009), 1181-1191.

[31] S. Cenberci and B. Peker, On the solutions of the equation $x^{2}+$ $19^{m}=y^{n}$, Notes on Number Theory and Discrete Mathematics 18 (2012), 34-41.

[32] S. Chowla, D. J. Lewis and Th. Skolem, The Diophantine equation $2^{n+2}-7=x^{2}$ and related problems, Proc. Amer. Math. Soc. 10 (1959), 250-257.

[33] J.H.E. Cohn, The diophantine equation $x^{2}+2^{k}=y^{n}$.II., Int. J. Math. Math. Sci., 22, (1999), 459-462.

[34] J.H.E. Cohn, The diophantine equation $x^{2}+2^{k}=y^{n}$, Arch. Math (Basel) 59 (1992), 341-344.

[35] J.H.E. Cohn, The diophantine equation $x^{2}+C=y^{n}$, Acta Arith. 65 (1993), 367-381.

[36] J.H.E. Cohn, The diophantine equation $x^{2}+C=y^{n} I I$., Acta Arith. 109 (2003), 205-206.

[37] E. Demirpolat, S. Cerberci and H. Senay, The Diophantine Equation $x^{2}+11^{2 k+1}=y^{n}$, International Mathematical Forum, 4, no. 6 (2009), 277-280.

[38] J. S. Ellenberg, Galois representations to $\mathbb{Q}$-curves and the generalized Fermat Equation $A^{4}+B^{2}=C^{p}$, Amer. J. Math. 126, 763-787 (2004).

[39] U. Fincke and M. Pohst, Improved methods for calculating vectors of short length in a lattice, including a complexity analysis, Math. Comp. 44 (1985), no. 170, 463-471.

[40] H. Godinho, D. Marques and A. Togbe, On the Diophantine equation $x^{2}+2^{\alpha} 5^{\beta} 17^{\gamma}=y^{n}$, Communications in Mathematics 20 (2012) 81-88.

[41] E. Goins, F. Luca and A. Togbe, On the Diophantine Equation $x^{2}+$ $2^{\alpha} 5^{\beta} 13^{\gamma}=y^{n}$, ANTS VIII Proceedings: A.J. van der Poorten and A. Stein (eds.), ANTS VIII, Lecture Notes in Computer Science 5011 (2008), 430-442. 
[42] K. Győry, I. Pink and Á. Pintér, Power values of polynomials and binomial Thue-Mahler equations, Publ. Math. Debrecen 65 (2004), 341-362.

[43] H. Hasse, Über eine diophantische Gleichung von RamanujanNagell und ihre Verallgemeinerung, Nagoya Math. J. 27 (1966), $77-102$.

[44] Y. Hu and M. Le, New Advances on the Generalized LebesgueRamanujan-Nagell Equation, Advances in Mathematics(China) 41 (2012), 385-396.

[45] M. Le, On the number of solutions of the generalized RamanujanNagell equation $x^{2}-D=2^{n+2}$, Acta Arith. 60 (1991), 149-167.

[46] M. Le, $A$ note on the Diophantine Equation $x^{2}+4 D=y^{p}$, Monatsh. Math. 116 (1993), 283-285.

[47] M. Le, On the number of solutions of the diophantine equation $x^{2}+D=p^{n}$, C. R. Acad. Sci. Paris Ser. I. Math. 317 (1993), $135-138$.

[48] M. Le, On the Diophantine equation $D_{1} x^{2}+D_{2}=2^{n+2}$, Acta Arith. 64 (1993), 29-41.

[49] M. Le, A note on the Generalized Ramanujan-Nagell Equation, J. of Number Theory $\mathbf{5 0}$ (1995), 193-201.

[50] M. Le, Some Exponential Diophantine Equations I. The Equation $D_{1} x^{2}-D_{2} y^{2}=\lambda k^{z}$, J. Number Theory 55 (1995), 209-221.

[51] M. Le, A Note on the Number of Solutions of the Generalized Ramanujan-Nagell Equation $D_{1} x^{2}+D_{2}=4 p^{n}$, J. of Number Theory 62 (1997), 100-106.

[52] M. Le, On the Diophantine equation $\left(x^{3}-1\right) /(x-1)=\left(y^{n}-\right.$ 1)/(y-1), Trans. Amer. Math. Soc. 351 (1999), 1063-1074.

[53] M. Le, On Cohn's conjecture concerning the diophantine equation $x^{2}+2^{m}=y^{n}$, Arch. Math. Basel 78 (2002), 26-35.

[54] M. Le, On the diophantine equation $x^{2}+p^{2}=y^{n}$, Publ. Math. Debrecen 63 (2003), 27-78.

[55] M. Le and H. Zhu, On some generalized Lebesque-Nagell equations, Journal of Number Theory 131 (2011), 458-469. 
[56] V. A. Lebesque, Sur l'impossibilité en nombres entierde l'equation $x^{m}=y^{2}+1$, Nouvelle Annales des Mathématiques (1) 9 (1850), $178-181$.

[57] A. K. Lenstra, H. W. Lenstra, Jr. and L. Lovász, Factoring polynomials with rational coefficients, Math. Ann. 261 (1982), 515-534.

[58] M.G. Leu and G.W. Li The Diophantine equation $2 x^{2}+1=3^{n}$, Proc. Amer. Math. Soc. 131 (2003) 3643-3645.

[59] W. Ljunggren, Über einige Arcustangensgleichungen die auf interessante unbestimmte Gleichungen führen, Ark. Mat. Astr. Fys. 29A (1943), No. 13.

[60] W. Ljunggren, On the diophantine equation $C x^{2}+D=y^{n}$, Pacific J. Math. 14 (1964), 585-596.

[61] F. Luca, On a diophantine equation, Bull. Austral. Math. Soc. 61 (2000), 241-246.

[62] F. Luca, On the equation $x^{2}+2^{a} 3^{b}=y^{n}$, Int. J. Math. Sci. 29 (2002), 239-244.

[63] F. Luca, Sz. Tengely, and A. Togbe, On the Diophantine equation $x^{2}+C=4 y^{n}$, Ann. Sci. Math. Quebec 33 (2009), 171-184.

[64] F. Luca and A. Togbe, On the Diophantine equation $x^{2}+7^{2 k}=y^{n}$, Fibonacci Quart. 54/4 (2007), 322-326.

[65] F. Luca and A. Togbe, On the Diophantine equation $x^{2}+2^{a} 5^{b}=$ $y^{n}$, Int. J. Number Th. 4/6 (2008), 973-979.

[66] M. Mignotte and B.M.M de Weger, On the equations $x^{2}+74=y^{5}$ and $x^{2}+86=y^{5}$, Glasgow Math. J. 38/1 (1996), 77-85.

[67] R. A. Mollin, A note on the Diophantine Equation $D_{1} x^{2}+D_{2}=$ $a k^{n}$, Acta Math. Acad. Paedagog. Nyhzi (N.S.) 21 (2005), 21-24.

[68] F.S. Abu Muriefah, F. Luca and A. Togbe, On the diophantine equation $x^{2}+5^{a} 13^{b}=y^{n}$, Glasgow Math. J 50 (2008), 175-181.

[69] T. Nagell, Sur l'impossibilité de quelques équations a deux indeterminées, Norsk. Mat. Forensings Skifter 13 (1923), 65-82.

[70] T. Nagell, Løsning till oppgave nr 2, Norsk. Mat. Tidsskrift 30 (1948), 62-64. 
[71] T. Nagell Contributions to the theory of a category of diophantine equations of the second degree with two unknowns, Nova Acta Reg. Soc. Upsal. IV Ser. 16, Uppsala 1955, pp. 1-38.

[72] T. Nagell The Diophantine equation $x^{2}+7=2^{n}$, Ark. Math. 4 (1960), 185-187.

[73] A. Pethö, H.G. Zimmer, J. Gebel and E. Hermann Computing all $S$-integral points on elliptic curves, Math. Proc. Cambridge. Phil. Soc 127 (1999) 383-402.

[74] I. Pink On the diophantine equation $x^{2}+2^{\alpha} 3^{\beta} 5^{\gamma} 7^{\delta}=y^{n}$, Publ. Math. Debrecen 70/1-2 (2007),149-166.

[75] I. Pink and Zs. Rábai On the diophantine equation $x^{2}+5^{k} 17^{l}=y^{n}$, Communications in Mathematics, 19, (2011), 1-9 .

[76] S. Ramanujan, Question 446, J. Indian Math. Soc. 5 (1913), 120, Collected papers, Cambridge University Press (1927), 327.

[77] N. Saradha and A. Srinivasan Solutions of some generalized Ramanujan-Nagell equations, Indag. Math. (N.S.) 17/1 (2006), 103-114.

[78] N. Saradha and A. Srinivasan Solutions of some generalized Ramanujan-Nagell equations via binary quadratic forms, Publ. Math. Debrecen 71/3-4 (2007), 349-374.

[79] N. Saradha and A. Srinivasan, Generalized Lebesgue-RamanujanNagell Equations, (2008), 207-223, Diophantine Equations, Editor: N. Saradha, Narosa Publishing House, New Delhi, India.

[80] A. Schinzel and R. Tijdeman, On the equation $y^{m}=P(x)$, Acta Arith. (1976), 31, 199-204.

[81] T.N. Shorey, A.J. van der Poorten, R. Tijdeman and A. Schinzel, Applications of the Gel'fond-Baker method to Diophantine equations, in: Transcendence Theory: Advances and Applications, Academic Press, London-New York, San Francisco, (1977), 59-77.

[82] T.N. Shorey, R. Tijdeman, Exponential Diophantine equations, Cambridge Tracts in Mathematics, 87. Cambridge University Press, Cambridge, 1986, x+240 pp.

[83] N. P. Smart, Determining the small solutions to $S$-unit equations, Math. Comp. 68 (1999), 1687-1699. 
[84] G. Soydan, On the Diophantine equation $x^{2}+7^{\alpha} 11^{\beta}=y^{n}$, Miskolc Mathematical Notes 13 (2012), 515-527.

[85] G. Soydan, M. Ulas and H. Zhu, ON THE DIOPHANTINE EQUATION $x^{2}+2^{a} 19^{b}=y^{n}$, Indian J. Pure Appl. Math. 43 (2012), 251-261.

[86] R. J. Stroeker and N. Tzanakis Solving elliptic diophantine equations by estimating linear forms in elliptic logarithms, Acta Arithmetica 67 (1994), 177-196.

[87] L. Tao, On the Diophantine equation $X^{2}+3^{m}=Y^{n}$, Integers: Electronic J. Combinatorial Number Theory 8 (2008), 1-7.

[88] L. Tao, On the Diophantine equation $x^{2}+5^{m}=y^{n}$, Ramanujan J. 19 (2009), 325-338.

[89] Sz. Tengely, On the Diophantine equation $x^{2}+a^{2}=2 y^{p}$, Indag. Math. (N.S.) 15 (2004), 291-304.

[90] Sz. Tengely, On the Diophantine equation $x^{2}+q^{2 m}=2 y^{p}$, Acta Arith. 127 (2007), 71-86.

[91] H. Virgolici, On the Exponential Diophantine Equation $x^{2}+D=$ $y^{n}:$ a brief survey, An. Univ. Spiru Haret. Ser. Mat.-Inform. 9 (2013), 45-54.

[92] B. M. M. de Weger, Algorithms for Diophantine equations, CWI Tract 65, Stichting Mathematisch Centrum, Amsterdam 1989.

[93] K. Wildanger, Über das Lösen von Einheiten- und Indexformgleichungen in algebraischen Zahlkr̈rpern, J. of Number Theory $\mathbf{8 2}$ (2000), 188-224.

[94] P. Xiaowei, The Exponential Lebesgue-Nagell Equation $x^{2}+p^{2 m}=$ $y^{n}$, Period. Math. Hungar. 67 (2013), 231-242.

[95] P. Yuan, On the number of the solutions of $x^{2}-D=p^{n}$, Sichuan Daxue Xuebao 35 (1998), 311-316.

[96] H. Zhu, A note on the Diophantine equation $x^{2}+q^{m}=y^{3}$, Acta Arith. 146 (2011), 195-202. 
Attila BÉRCZES,

Institute of Mathematics

University of Debrecen

H-4010 Debrecen, P.O. Box 12

Hungary Email: berczesa@science.unideb.hu

István PINK Institute of Mathematics

University of Debrecen

H-4010 Debrecen, P.O. Box 12

Hungary Email: pinki@science.unideb.hu 